\title{
Sinus tarsi approach for displaced, Intra-articular Calcaneal Fractures
}

\author{
Authors

\section{Dr Manzoor Ahmad Halwai ${ }^{1}$, Dr Mudasir Malik ${ }^{2}$, Dr Mohammad Yaseen Rather ${ }^{3}$,} \\ Dr Bashir Ahmad Mir ${ }^{4}$, Dr Asif Sultan ${ }^{5}$ \\ ${ }^{1}$ Professor and Head, Department of Orthopaedics, GMC Srinagar \\ ${ }^{2}$ Lecturer, Department of Orthopaedics, GMC Srinagar \\ ${ }^{3}$ Registrar, Department of Orthopaedics, GMC Srinagar \\ ${ }^{4}$ Professor, Department of Orthopaedics, GMC Srinagar \\ ${ }^{5}$ Assistant Professor, Department of Orthopaedics, GMC Srinagar
}

\section{Introduction}

The Calcaneus is the largest bone of the foot and is the major weight bearing osseous structure of the foot ${ }^{1}$. Calcaneal fracture fixation is regarding as one of the serious orthopaedic problems fraught with many complications ranging from osseous, soft tissue related and morbidity sometimes very high $^{1,2,3}$.

Calcaneal fractures make up to 2 percent of all fractures, with a displacing intra articular fractures comprising 60 percent to 75 percent of these injuries $^{2,4}$. There is a substantial economic implications associated with these fractures with as many 20 percent of people remain functionally incapacitated for up to three years after injury ${ }^{5}$.

With introduction of calcaneal plates in 1980, open reduction and internal fixation became standard $^{5,6}$. But these were associated with high complication rates ranging from wound dehiscence, osteomyelitis, personal tendinitis, sural nerve injury, fibular abutment, calcaneocuboid arthritis, stiff foot and toes, weak gastrosoleus complex, fixed flat foot, chronic heal pain and complex regional pain syndrome $e^{3,7,8,9}$.
Wound dehiscence and infection are particularly unique to this procedure ${ }^{2,6}$.

Conservative management has its own problems like subtalar osteoarthritis, subfibular impingement, complex regional pain syndrome and it seems logical to restore calcaneus to its anatomical contours ${ }^{7,10,11}$.

Minimally invasive osteosynthesis using sinus Tarsi approach can be a procedure between open reduction internal fixation using lateral extensive approach and conservative management ${ }^{1,3,4,12}$. It's advantages are restoration of anatomical contours of calcaneum like calcaneal height, width, restoration of calcaneal length, visualisation of posterior facet and no need for extensive lateral approach and no guilt of not restoring anatomy as in conservative treatment ${ }^{13,14}$.

\section{Materials and Methods}

The study was conducted at bone and joint hospital barzullah Srinagar from January 2015 to January 2017.

The inclusion criteria were as follows:

\section{Age group 20 to 60}


2. Duration of injury within 3 weeks of trauma

3. Sanders types II, III, IV

4. Closed calcaneal fractures

\section{Exclusion criteria}

1. Age less than 18 and greater than 60

2. Extra articular fractures

3. Sanders type I fractures

4. Associated major fractures which hampers postoperative rehabilitation

5. Patients with associated neurological disorders

6. Bilateral calcaneal fractures

After admission to hospital all necessary investigations were carried out, particularly CT SCAN with coronal and axial images were analysed to determine displacement of the articular surface of posterior facet and number and location of articular fracture fragments. Fractures were classified according to SANDERS CLASSIFICATION for calcaneal fractures.

\section{Operative technique}

All patients were operated under Spinal Anaesthesia and Torniquet control. Patients were drapped and operated in lateral decubitus position. Image intensifier was kept standby and operated by expert Radiographer who is proficient in taking all views of Calcaneus.

A $2 \mathrm{~cm}$ incision was given over sinus tarsi. The deep soft tissues were divided carefully to protect the Sural Nerve. Posterior facet was exposed and elevated under vision. Provisional fixation was done with $\mathrm{K}$ wires. Once articular surface was restored, a subchondral screw was passed from lateral to medial in posterior to anterior direction directed towards sustenticulam tali. Final screw position is confirmed by taking proper radiographic views.

Next steinmen pin was passed from medial to lateral in calcaneal tuberosity with equal lengths of pin on either side of tuberosity. Traction was applied on pin and at same time valgus stress was applied to correct varus deformity. While maintaining traction on pin and calcaneal height, length and heel postioned restored, provisional fixation done with $\mathrm{K}$ wires. And finally two parallel screws were passed from posterior to anterior part of calcaneum for fixation, and its position confirmed on image intensifier. Wound was closed and below knee cast applied for 3 months with absolute non weight bearing on the involved side.

Postoperative care Final assessment was done at nine months with following considerations

A. Clinical assessment was according to American Orthopaedic foot and Ankle society (AOFAS) hind foot score $\mathrm{s}^{1,2,5,6,15,16,17}$. This scoring was graded as follows:

Excellent: More than 90

Good: 80-90

Fair: 70-79

Poor: Less than 69

B. Radiographic outcome

\section{Observations and Results}

This study was conducted on 60 patients from January 2015 to January 2017 with minimum follow up of 9 months and maximum of 29 months with average follow up of 16 months Age distribution Most patients were in age group of 30-39 years $(50 \%)$, second commonest was age group 40-49 years. Youngest patient was 20 years and eldest was 60 years.

We operated on 60 percent of male patients and 40 percent of female patients. we operated on left side in 57 percent of patients and on right side in 43 percent of patients.

We had 17 percent Sanders type II, 47 percent were Sanders type III, and 36 percent were type IV. Injury to surgery interval ranged from 3 days to 12 days, average injury interval was 6.6 days. Surgery time was average of 45 minutes. About 90 percent of patients were discharged next day after operation. All patients were kept nonweight bearing for a period of 12 weeks.

\section{Outcome scores}

We used AOFAS scale for clinical evaluation of the ankle and hind foot. We achieved excellent 
results in 30 percent and good in 57 percent and fair in 13 percent of cases.

Fair results in our study were in patients where complete anatomic fixation was not achieved $(7 \%)$.

\section{Complications}

We had no case of wound infection or skin related problems, No case of non union.

We had complication in the form of not able to achieve anatomical contours which was most of times a surgeons inability to follow surgical steps. We had decreased heal height in 10 patients, heel broadening in 7 patients and subfibular impingement in 5 patients which was symptomatic in 1 patient only.

\section{Discussion}

Calcaneal fractures are devastating injuries with lot of morbidity associated both with conservative and operative treatment ${ }^{1,18}$.

Restoration of calcaneus to its pre-fracture contours is the goal of operative treatment ${ }^{19,20}$. Restoration of calcaneal height, length, articular facet congruence is important for optimal results but one cannot ignore soft tissue respect, and the punishment which patient and operating surgeon goes through, if soft tissue problems arise ${ }^{21,22}$.

Lateral extensible approach is used for open reduction internal fixation of intra articular calcaneal fractures ${ }^{23,24}$. But it has many tormenting complications especially of soft tissues and wound complications reported as high as $50 \%$ by some studies ${ }^{12,25,26}$. Lateral extensible approach may cause lateral flap necrosis due to poor blood supply, may be associated with nerve injuries (sural nerve injuries) and infection ${ }^{27,38,29}$. Conservative treatment has its indications but it appears logical to restore calcaneus to its pre injury parameters when they are disturbed by fracture. There are many theories to explain poor results of non operative management most important being non anatomical reduction ${ }^{18,30}$. Minimally invasive method using sinus Tarsi approach is intermediate between conservative and operative reduction with lateral extensive $\operatorname{approach}^{1,9,16}$.

It's modus operandi of action is to restore calcaneal dimensions with focus on restoration of calcaneal height, length, heal position and operative reduction of posterior facet not by indirect method but under supervision of surgeons eye $^{2,16,18}$. These things cannot be achieved by conservative treatment and this is where it takes precedence compared to conservative treatment. We don't need extensive dissection to achieve reduction and avoid many complications of lateral extensive approach ${ }^{19,31}$.

It takes less time for operating using this technique, less blood loss, no incidence of nonunion, low complication rate, bone graft was never used, And drastically reduced infection rate, no painful and ugly scars ${ }^{32,33}$.

\section{Summary and Conclusion}

Minimally invasive calcaneal fracture management is excellent option for operative treatment of calcaneal fractures and is excellent option comparable to open reduction and internal fixation. It has fewer complications and is almost devoid of soft tissue problems.

\section{References}

1. Abidi NA, Dhawan S, Gruen GS, Vogt MT, Conti SF (1998). Wound healing risk factors after open reduction and internal fixation of calcaneal fractures. Foot Ankle Intl 19:856861.

2. Harvey EJ, Grujic L, Early JS, Benirschke SK, Sangeorzan BJ (2001). Morbidity associated with ORIF of intraarticular calcaneal fractures using a lateral approach. Foot Ankle Intl 22:868-873.

3. Stulik J, Stehlik J, Rysavy M, Wozniak A (2006). Minimally Invasive treatment of intra-articular fractures of the calcaneum. J Bone Joint Surg B 88:1634-1641.

4. Westphal T, Piatek S, Halm J, Schubert, Winckler. Outcome of surgically treated intraarticular calcaneus fractures-SF-36 
compared with AOFAS and MFS. Acta Orthop. 2004;75(6):750-5.

5. Brunner A, Müller J, Regazzoni P, Babst R. Open reduction and internal fixation of OTA type C2-C4 fractures of the calcaneus with a triple-plate technique. The Journal of Foot and Ankle Surgery. n.d.;51(3):299307.

https://doi.org/10.1053/j.jfas.2012.01.011.

6. Clare MP, Crawford WS. Managing complications of Calcaneus fractures. Foot Ankle Clin. 2017;22(1):105-16. https://doi.org/10.1016/j.fcl.2016.09.007.

7. Yeap EJ, Rao J, Pan $\mathrm{CH}$, Soelar SA, Younger AS. Is arthroscopic assisted percutaneous screw fixation as good as open reduction and internal fixation for the treatment of displaced intra-articular calcaneal fractures?

8. Phisitkul P, Sullivan JP, Goetz JE, Marsh JL. Maximizing safety in screw placement for posterior facet fixation in calcaneus fractures: a cadaveric radio-anatomical study. Foot \& Ankle International. 2013;34(9):1279-85. https://doi.org/10.1177/1071100713487182.

9. Bussewitz BW, Hyer CF. Screw placement relative to the calcaneal fracture constant fragment: an anatomic study. J Foot Ankle Surg. 2015;54(3):392-4.

10. Reinhardt S, Martin H,Ulmar B, Döbele S, Zwipp H, Rammelt S, et al. Interlocking nailing versus interlocking plating in intraarticular calcaneal fractures: a biomechanical study. Foot Ankle Int. 2016;37(8):891-7. https://doi.org/10.1177/1071100716643586.

11. Abdelgawad AA, Kanlic E. Minimally invasive (sinus tarsi) approach for open reduction and internal fixation of intraarticular calcaneus fractures in children: surgical technique and case report of two patients. J Foot Ankle Surg. 2015;54 (1):135-9.
12. Lu D, Zhu SY, Yang J, Chen H. Sun LJ. IA comparison of two minimally invasive procedures for intra-articular displaced calcaneal fractures in older children. Acta Orthop Belg. 2016;82(4):779-86.

13. Al-Mudhaffar M, Prasad C, Mofidi A. Wound complications following operative fixation of calcaneal fractures. Injury. 2000;31(6):461-4.

14. Benirschke SK, Kramer PA. Wound healing complications in closed and open calcaneal fractures. J Orthop Trauma. 2004;18(1):1-6.

15. Folk JW, Starr AJ, Early JS. Early wound complications of operative treatment of calcaneus fractures: analysis of 190 fractures. $\mathbf{J}$ Orthop Trauma. 1999;13(5):369-72.

16. Harvey EJ, Grujic L, Early JS, Benirschke SK, Sangeorzan BJ. Morbidity associated with ORIF of intra-articular calcaneus fractures using a lateral approach. Foot Ankle Int. 2001;22(11):868-73.

17. Yeo J, Cho H, Lee K. Comparison of two surgical approaches for displaced intraarticular calcaneal fractures: sinus tarsi versus extensile lateral approach. BMC Musculoskelet Disord. 2015;16(1):63.

18. Xia S, Lu Y, Wang H, Wu Z, Wang Z. Open reduction and internal fixation with conventional plate via L-shaped lateral approach versus internal fixation with percutaneous plate via a sinus tarsi approach for calcaneal fractures - a randomized controlled trial. Int J Surg. 2014;12(5):47580.

19. Wu Z, Su Y, Chen W, Zhang Q, Liu Y, Li M, Zhang Y. Functional outcome of displaced intra-articular calcaneal fractures. J Trauma Acute Care Surg. 2012;73(3):74351.

20. Weber M, Lehmann O, Sagesser D, Krause F. Limited open reduction and internal fixation of displaced intra-articular fractures of the calcaneum.J Bone Joint Surg Br Vol. 2008;90-B(12):1608-16. 
21. Phan K, Mobbs R. Systematic reviews and meta-analyses in spine surgery, neurosurgery and orthopedics: guidelines for the surgeon scientist. J Spine Surg. 2015;1(1):19-27.

22. Higgins JP, Thompson SG. Quantifying heterogeneity in a meta-analysis. Stat Med. 2002;21(11):1539-58.

23. Shuler FD, Conti SF, Gruen GS, Abidi NA. Wound-healing risk factors after open reduction and internal fixation of calcaneal fractures. Orthop Clin N Am. 2001;32(1):187-92.

24. Abdelgaid SM. Closed reduction and percutaneous cannulated screws fixation of displaced intra-articular calcaneus fractures. Foot Ankle Surg. 2012;18(3):164-79.

25. Biggi F, Fabio SD, D’Antimo C, Isoni F, Salfi C, Trevisani S. Percutaneous calcaneoplasty in displaced intraarticular calcaneal fractures. J Orthop Traumatol. 2013;14(4):307-310a.

26. Harvey EJ, Grujic L, Early JS, et al. Morbidity associated with ORIF of intraarticular calcaneus fractures using a lateral approach. Foot Ankle Int 2001;22:868-73.

27. Shuler FD, Conti SF, Gruen GS, Abidi NA. Wound-healing risk factors after open reduction and internal fixation of calcaneal fractures: does correction of Bohler's angle alter outcomes? Orthop Clin North Am 2001;32:187-92.

28. Brauer CA, Manns BJ, Ko M, et al. An economic evaluation of operative compared with nonoperative management of displaced intra-articular calcaneal fractures. J Bone Joint Surg Am 2005;87:2741-9.

29. Pozo JLL, Kirwan EO, Jackson AM. The long-term results of conservative management of severely displaced fractures of the calcaneus. J Bone Joint Surg $\mathrm{Br}$ 1984;66:386-90.

30. Crosby LA, Fitzgibbons T. Intraarticular calcaneal fractures. Results of closed treatment. Clin Orthop Relat Res 1993;290:47-54.

31. Arastu M, Sheehan B, Buckley R. Minimally invasive reduction and fixation of displaced calcaneal fractures: surgical technique and radiographic analysis. Int Orthop 2014;38:539-45.

32. Labbe JL, Peres O, Leclair O, et al. Minimally invasive treatment of displaced intra-articular calcaneal fractures using the balloon kyphoplasty technique: preliminary study. Orthop Traumatol Surg Res 2013;99:829-36.

33. Su Y, Chen W, Zhang Q, et al.. Bony destructive injuries of the calcaneus: longterm results of a minimally invasive procedure followed by early functional exercise: a retrospective study. BMC Surg. 2014;14:19. 